\title{
Introduction to Laser Ablation Video Supplement
}

\author{
Gene H. Barnett, MD, MBA, ${ }^{1}$ Andrew E. Sloan, MD, ${ }^{2}$ and Claudio E. Tatsui, MD ${ }^{3}$
}

${ }^{1}$ The Cleveland Clinic, Brain Tumor Institute; '2Department of Neurological Surgery, University Hospital-Case Medical Center, Cleveland, Ohio; and ${ }^{3}$ Department of Neurosurgery, MD Anderson Cancer Center, Houston, Texas

\begin{abstract}
Laser ablation (also known as laser interstitial thermal therapy [LITT]) has emerged as an important new technology for treating various disorders of the brain and spine. As with any new or emerging technology, there is a learning curve for its optimal use, and video tutorials can be important learning tools to help bridge gaps in knowledge for those who wish to become more familiar with laser ablation. In this special supplement to Neurosurgical Focus, videos illustrate laser ablation's use in the treatment of epilepsy and failed radiosurgery, as well as technical aspects of performing these procedures in eloquent brain and in the spine. We hope that these videos will enable you to enhance your understanding of the evolving use of laser ablation for disorders of the brain or spine. It is the editors' sincere hope that this will be helpful either in your own practice or in determining whether to refer to a neurosurgical colleague experienced in this field.
\end{abstract}

KEYWORDS laser ablation; laser interstitial thermal therapy; LITT; surgical technique; video 